\title{
Medición de Tiempos en un Sistema de Distribución bajo un Estudio de Métodos y Tiempos
}

\author{
Gustavo R. Henríquez-Fuentes ${ }^{(1) \star}$, Diego A. Cardona ${ }^{(2)}$, Jesús A. Rada-Llanos ${ }^{(3)}$ y Nilka R. Robles ${ }^{(4)}$ \\ (1) Univ. de la Costa, Barranquilla, Colombia. (e-mail: ghenriqu4@cuc.edu.co) \\ (2) Universidad Libre de Cartagena, Colombia. (e-mail: diego.cardona@unilibrectg.edu.co) \\ (3) Programa de Administración de Empresas, Univ. Autónoma del Caribe. (e-mail: jrada@uac.edu.co) \\ (4) Univ. de la Guajira, Facultad Ciencias Económicas y Administrativas. (e-mail: nrobles@uniguajira.edu.co)
}

* Autor a quien debe ser dirigida la correspondencia

Recibido Mar. 8, 2018; Aceptado May. 23, 2018; Versión final Jul. 21, 2018, Publicado Dic. 2018

\begin{abstract}
Resumen
El objetivo del presente artículo es, diseñar una herramienta para medir tiempos en el sistema de distribución de una comercializadora y mejorar su sistema de distribución. La metodología, de tipo cuantitativo, emplea estadística descriptiva para analizar la gestión de las rutas de la empresa y conocer su situación actual. Basada en la distribución de una comercializadora de productos importados, la investigación se desarrolló por espacio de 15 días, permitiendo identificar prácticas inadecuadas en las rutas de distribución. Como resultado se propone un modelo gráfico para la distribución de mercancías denominado METDE. Se plantea también una metodología para la planificación de rutas óptimas de distribución basada en el estudio de métodos y tiempos de las rutas entre la empresa y sus clientes. Se detectaron las variables relevantes del contexto logístico que afectan los tiempos y el cumplimiento en los horarios de entregas de los pedidos.
\end{abstract}

Palabras clave: cadena de suministro; medición en distribución; logística de distribución; métodos Cuantitativos; centro de distribución CEDI.

\section{Measurement for a Distribution System under a Study of Methods and Times}

\begin{abstract}
The objective of this article is to design a tool to measure times in the distribution system of a marketer and improve its distribution system. The methodology is of quantitative type uses descriptive statistics to analyze the management of the routes of the company and to know its current situation. Based on the distribution of a company of imported products, the research was carried out for 15 days, allowing the identification of inappropriate practices in the distribution routes. As a result, a graphic model for the distribution of goods called METDE is proposed, Also, a methodology for the planning of optimal distribution routes based on the study of methods and times of the routes between the company and its customers is developed. The most important variables of the logistical context that affected the times and compliance in the delivery schedules of the orders were identified.
\end{abstract}

Keywords: supply chain; distribution logistics; quantitative methods; CEDI distribution center. 


\section{INTRODUCCIÓN}

El sistema de gestión logística se encarga de abastecer oportunamente a la empresa, mientras gestiona la rotación eficiente de los inventarios en el almacén, coordinando al tiempo, las tareas del sistema de operaciones o producción del producto o servicio y la manera como se distribuye y se dispone en el punto de venta, gestionando, además, la satisfacción y el servicio al cliente. La programación de las tareas es fundamental, ya que el sistema logístico, debe funcionar según lo programado para que los materiales se administren de manera inteligente en el sitio, sin encontrar la presencia de materiales excesivos (Mydin et al., 2014). No hay cabida para el desperdicio de recursos en un proceso que compromete muchas funciones.

La logística atraviesa todos los procesos de una empresa, siendo considerada como un proceso transversal que integra las operaciones de varias empresas en lo que se denomina Cadena de Suministro (en adelante CDS). La logística, permite a las directivas organizar los procesos para proveerse y movilizar mercancías, acopiar información del mercado y ajustar los tiempos de entrega y los costos de transacción de las operaciones; desarrollando procesos estratégicos y misionales como servicio al cliente, gestión de inventarios, producción, abastecimiento, distribución y almacenamiento (Henríquez et al., 2018; Henríquez, 2013). La CDS exige fortalecer las relaciones entre sus miembros, desde el proveedor inicial, que entrega algo procesado o no, hasta un cliente que puede a su vez, ser proveedor de otro hasta entregar a un cliente final que consume ese algo transmitido y transformado en cadena; reconociendo que el fortalecimiento con otros miembros y las relaciones de largo plazo con proveedores tiene efectos significativos en el rendimiento de la empresa (Vélez, 2014; Prajogo y Olhager, 2012). El mayor grado de integración con proveedores y clientes está asociado fuertemente con la mejora del rendimiento (Frohlich y Westbrook, 2001). En la CDS prevalece la colaboración, la confianza y la acción de muchos miembros con objetivos comunes. En la actualidad, debido a las presiones de grupos ambientalistas, personas naturales o el estado en cuanto a cuidado del medio ambiente, el diseño de la CDS exige de la participación de los Stakeholders (Rajeev et al., 2017).

En el entorno empresarial uno de los factores críticos es el rendimiento de la CDS, siendo considerada la medición como fundamental para gestionar eficientemente (Hasan et al., 2016). Algunas medidas de desempeño se ven afectadas en términos de tiempo, costo, fiabilidad y flexibilidad por factores como la infraestructura, aduanas, transporte internacional, la competencia logística y el seguimiento de la puntualidad (Jhawar et al., 2014). El gerente debe procurar mediciones versátiles en las áreas de abastecimiento, producción y distribución, así como evaluar el estado de cohesión con las demás empresas que integran la CDS; ya que los sistemas de control operativo y de gestión deben ir más allá de la medición de objetivos financieros (Kaplan y Norton, 1996). La efectividad de los procesos debe ser medida periódicamente, ya que sirve como una herramienta para la identificación de fallas, logros y reajuste de metas (Cardona et al., 2017). Dentro de la CDS se desarrollan tres procesos importantes considerados eslabones logísticos, son estos, el abastecimiento, producción-operación y el proceso de distribución. En el tercer proceso de la logística, la distribución, es donde se concentró el esfuerzo de esta investigación. La logística de distribución o logística de salida, es una estrategia para atender los pedidos de los clientes por medio de una CDS en condiciones de calidad y tiempo, en un lugar y a un costo; este proceso representa aproximadamente el $25 \%$ del precio final de los bienes (Gómez y Correa, 2011; Arrubla, 2011).

Para llevar los productos desde la empresa hasta los canales de distribución o puntos de venta, se requiere de un sistema de transporte eficiente, integrado y comunicado entre las partes, para monitorear constantemente las mercancías, los tiempos de entrega, costos incurridos, y la manera en que es gestionada la información a lo largo del proceso. La empresa debe medir su sistema de distribución para conocer su desempeño y controlarlo. La información deberá fluir a través de los actores del sistema de distribución, definiendo claramente los objetivos a medir del sistema en sus diversos actores tales como: 1) La empresa y sus operaciones; 2) los canales de distribución; 3) clientes; 4) los clientes de mis clientes (Cardona et al., 2017). La CDS deberá medir sus operaciones para proceder a controlar sus procedimientos y alcanzar resultados en cada eslabón, entre estos, el de distribución dónde más contacto se tiene con el cliente. Muchos son los aportes realizados a la gestión de la CDS a partir de modelos que proponen la cooperación de los miembros de la cadena (Balza et al., 2017). Otros, se centran en la estrategia de negociación entre compradores y vendedores, donde toma importancia el poder de negociación de los actores y variables como el precio y tiempo de espera (lead time) que inciden en el desempeño de la CDS (Yu et al., 2015). Otros aportes estiman las bondades del outsourcing o la administración del riesgo de las operaciones para el buen desempeño de la CDS, así como la gestión basada en servicio al cliente, la competencia de servicios y el bienestar de los consumidores (Wang et al., 2015).

Un modelo que sirve para medir los procesos logísticos es el SCOR, el cual sirve como herramienta de diagnóstico a los procesos globales, permitiendo que las compañías mejoren el rendimiento de sus sistemas en cuanto a las mejores prácticas (Amaya et al., 2014). El cumplimiento de los tiempos de entrega, la calidad de pedido, la entrega perfecta recibida y la puntualidad de los despachos son indicadores que permiten 
conocer el desempeño logístico de las empresas (Martínez et al., 2017). Otros criterios como la responsabilidad, flexibilidad, confiabilidad, elementos tangibles, garantía, empatía, rentabilidad, costos, bienes y la utilización de recursos, son medidos a través de una serie de métricas que permiten conocer el desempeño de la CDS en áreas como las operaciones, el servicio al cliente, y la gestión corporativa (Cho et al., 2012). La medición permite conocer el desempeño del sistema de distribución. El diseño de las rutas de los vehículos es de las funciones operativamente hablando, más crítica en lo que se refiere al transporte (Gutiérrez et al. 2007). El costo, tiempo, riesgo y versatilidad, son las variables por estudiar en la utilización de cualquier clase de transporte (Vélez, 2014).

Actualmente se cuenta con muchas herramientas entre una gran diversidad de software que están disponibles para diseñar rutas de distribución (Baker, 2001). Estas herramientas denominadas como Vehicle Routing Problem (VRP) están diseñadas para optimizar los problemas de ruteo de vehículos y resolverlos en la medida que integran gráficas de información (Kolli et al., 1993). Los Sistemas de Información satelital se orientan al tratamiento de información sobre un sector geográfico, capturando datos por tecnología GPS y/o a través de la disponibilidad de satélites de observación de alta resolución para aplicaciones civiles; utilizan información geográfica de un sistema y algoritmos iterativos autoajustables (Casanava, 2010; Chen et al., 2016; Ying y Lin, 2018). Las herramientas para la gestión de flotas de vehículos permiten monitorear en tiempo real aquellos parámetros considerados más importantes en la conducción y operatividad de la distribución urbana de mercancías, facilitando la eficaz gestión de rutas de reparto (León et al., 2016).

La gestión de distribución hace referencia al diseño para la localización de instalaciones, el enrutamiento y la programación de vehículos y las tripulaciones para cumplir con los objetivos trazados para la entrega de mercancías (Kolli et al., 1993). Para la solución a los problemas en el diseño de rutas, existe la propuesta de un algoritmo para problemas de rutas de vehículos (VRP por sus siglas en inglés) buscando el equilibrio entre los costos del viaje y la satisfacción del cliente (Kovacs et al., 2015). Otros modelos de enrutamiento basan su eficiencia en el consumo de combustible y las emisiones de dióxido de carbono para el enrutamiento de vehículos basado en la potencia requerida para su desplazamiento (Suarez et al., 2018). Se cuenta actualmente con una gran variedad de herramientas y modelos para la organización de la distribución.

La investigación que se describe en este trabajo tiene como objetivo el diseño de una herramienta para la medición de tiempos para la logística de salida, contribuyendo a la gestión del transporte y la medición en logística de distribución en lo que se refiere al cumplimiento con los clientes, teniendo en cuenta que la logística y las operaciones sirven como entrada coordinada e integrada para crear valor a los clientes en los canales de comercialización (Kozlenkova et al., 2015). Se realizó a partir de un análisis de operaciones de una empresa comercializadora de productos importados en Barranquilla-Colombia, con un sistema de distribución inapropiado, que presenta errores en la asignación de los pedidos y genera sobrecostos a la empresa e insatisfacción en sus clientes principales por el incumplimiento en algunas entregas. Se busca estimular la competitividad de la logística en Colombia y sea aplicado en cualquier empresa a nivel nacional o Internacional para ordenar su estrategia distribución. El estudio centra sus propósitos en el sistema de transporte, ya que, en la logística, los costos generales se ven afectados principalmente por la operación de transporte (Balza et al., 2017).

Su importancia además recae en el nivel estratégico, dónde se toman decisiones en logística que influyen en toda la organización, orientadas al objetivo final de la empresa, las cuales pueden cambiar debido a las tendencias del mercado (Voznenko y Román, 2015). La logística requiere la aplicación de herramientas prácticas que permitan conocer el estado de los eslabones de la CDS, por lo cual, se propone una herramienta basada en tecnologías de la información de uso común actualmente como es el aplicativo excel. Las tecnologías de la información y la comunicación permiten que la información se comparta, integrándola para tener un efecto positivo en la coordinación de los procesos (Prajogo y Olhager, 2012). El aporte de esta investigación se remite a la utilización de un aplicativo que toma en cuenta la medición de tiempos entre el centro de operaciones (en adelante CEDI) y los clientes, proponiendo el reordenamiento de las rutas con el cumplimiento de los tiempos, como objetivo principal.

\section{MATERIALES Y MÉTODOS}

El estudio se ha realizado bajo las bondades de los métodos cuantitativos, analizando el sistema de distribución de una comercializadora de productos importados. En la distribución, se identificaron fallas como el incumplimiento de los tiempos de entrega y sobrecostos generados por la baja eficiencia del sistema de distribución, por ejemplo, visitando a un mismo cliente dos veces en el día para lograr la entrega. Se realizó en la ciudad de Barranquilla y su área metropolitana por un espacio de 15 días, midiendo los tiempos empleados entre el centro de distribución y los principales clientes de la empresa al momento de realizar la entrega de los productos. La medición se realizó in-situ, por lo cual, fue necesario el acceso a los vehículos contratados por la empresa para cubrir sus rutas. 
Los métodos cuantitativos son herramientas y técnicas empleadas para la búsqueda de soluciones a problemas por medio del trabajo conjunto de la matemática, la estadística, la investigación de operaciones y la computación; ayudan a tomar decisiones, reduciendo el azar, riesgo, y sesgos del método intuitivo; ofreciendo certeza y confianza que se puede tener en las matemáticas y la estadística para estudiar variables (Falcon et al., 2016; Henríquez et al., 2016). Se propone una metodología cuantitativa para identificar, analizar y estudiar los factores que afectan la distribución de la empresa, apoyando así, la toma de decisiones en las operaciones, reduciendo el riesgo de estas. La metodología consiste en unos pasos que orientan la realización de un análisis actual de la CDS y la(s) ruta(s) de distribución, un análisis crítico del sistema de distribución, y una propuesta de mejoramiento de la(s) ruta(s).

\section{Medición de la distribución}

El paso 1, denominado situación actual, consiste en conocer la situación actual a partir del diseño de la CDS, desde los proveedores hasta los clientes (figura 1). Se utilizó una adaptación del diseño de una CDS de tipo "1 bodega N clientes" para entender la dinámica del sistema logístico de la empresa bajo estudio (Vidal et al., 2004). Los encargados del área de logística deberán medir el tiempo de espera (lead time) y la estructura de costos del proceso, desde proveedores hasta clientes, midiendo el sistema de abastecimiento; almacenamiento y operaciones y la distribución, tal como se indica en la CDS de la empresa (figura 1).

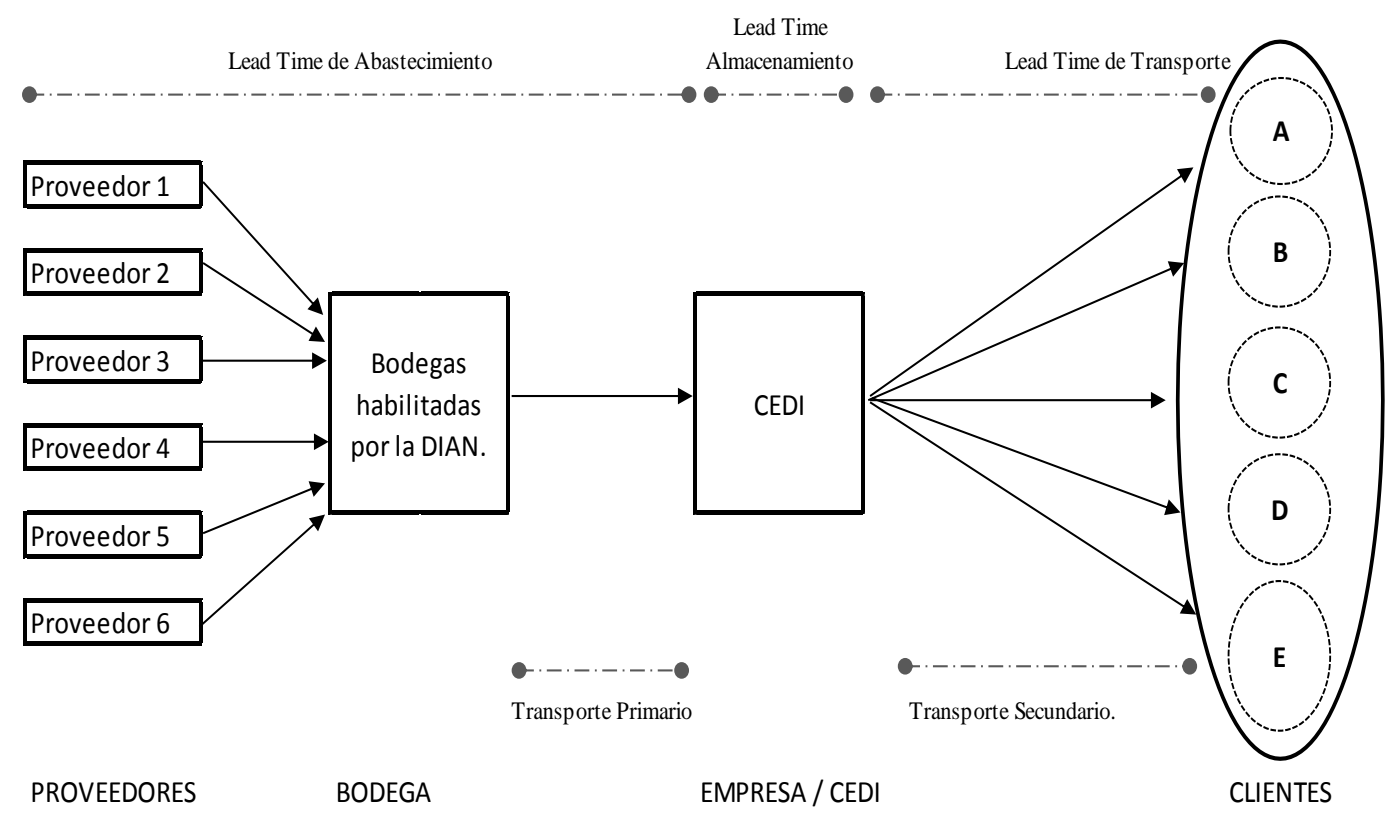

Fig. 1: Cadena de Abastecimiento con un CEDI y N clientes. Adaptada de Vidal, Londoño y Conteras (2004).

Se entiende a partir del diseño de la CDS, que la empresa recibe y nacionaliza sus mercancías en las bodegas o zonas habilitadas por la DIAN (Dirección de Impuestos y Aduanas Nacionales-Colombia), utilizando los servicios de operadores logísticos e intermediarios aduaneros para realizar los movimientos y trámites de la mercancía en los tres procesos logísticos (abastecimiento, operaciones-producción y distribución). El sector de servicios agiliza las operaciones empresariales, debido al grado de especialización de los operadores de servicios en cada campo. Se observó que la empresa realizó pedidos a 6 proveedores, contratando el servicio de transporte y operadores logísticos para el proceso de transporte primario (Abastecimiento) y transporte secundario (distribución) de mercancías. El principal problema en la distribución se refiere al incumplimiento en los tiempos de entrega a los clientes.

El Análisis crítico del estado de las rutas (Paso 2), es presentado a partir de la Tabla 1, en la que se pueden observar las mediciones de tiempo de la distancia entre el origen Y (CEDI- Empresa) y los destinos (clientes). Para este estudio se escogió de una muestra de 20 clientes, los 5 más representativos para la empresa en lo que se refiere a frecuencia de Distribución; midiendo por espacio de 15 días los tiempos empleados en los recorridos. Una vez medidas las distancias, se identificaron aspectos negativos o variables encontradas en las rutas, las cuales, afectan los tiempos de entrega.

En la tabla 1, se registraron los tiempos que toma el recorrido entre el punto $\mathrm{Y}$ al destino $\mathrm{A}, \mathrm{y}$ todos los demás destinos, tomando como origen de la(s) ruta(s) el punto Y (CEDI o empresa) 
Tabla 1: Medición de Tiempos desde CEDI a Destinos.

\begin{tabular}{|c|r|r|r|l|l|l|l|l|l|l|l|l|r|r|r|r|}
\hline Origen /Destino & D1 & D2 & D3 & D4 & D5 & D6 & D7 & D8 & D9 & D10 & D11 & D12 & D13 & D14 & D15 & Promedio \\
\hline Y-A & 11 & 12 & 9 & & & 11 & & 10 & & 9 & 8 & 10 & & & 9 & 9,89 \\
\hline Y-B & & & & 45 & & & 48 & & & & & & 53 & & 48,67 \\
\hline Y-C & 68 & 65 & 69 & & & & & & 62 & & & 67 & & 63 & 65,67 \\
\hline Y-D & & & & & 90 & & & 88 & 89 & & 77 & & & 83 & 85,40 \\
\hline Y-E & & & & & & 95 & 98 & & & 100 & & & 105 & & 106 & 100,80 \\
\hline A-B & & & 43 & & 42 & & & & & & & & & 37 & 40,67 \\
\hline A-C & & & & & & 54 & & 59 & & 51 & & & 58 & & 55,50 \\
\hline A-D & 74 & & 79 & & & & & & 78 & 68 & & 75 & & & 75 & 74,83 \\
\hline A-E & & 89 & & & 88 & & & & & & 91 & & & & 89,33 \\
\hline B-C & & & 13 & & & & & & & & & & 14 & 17 & 15 & 14,75 \\
\hline B-D & 34 & & & 36 & & & & & & & & & & & 35,00 \\
\hline B-E & 50 & & & & & & 49 & & & & & & & & & 49,50 \\
\hline C-D & & 22 & & & & 19 & & & & & & 18 & & & & 19,67 \\
\hline C-E & & & & & & & 31 & 33 & 36 & & & & & & 39 & 34,75 \\
\hline D-E & & & & & 18 & & & & 15 & 14 & & & 12 & & & 14,75 \\
\hline
\end{tabular}

Posteriormente, se registraron los tiempos desde el destino $\mathrm{A}$ hasta los demás destinos; desde $\mathrm{B}$ a los demás destinos; y así de manera sucesiva desde C, D y E hasta los demás destinos. Este procedimiento se realizó diariamente para obtener el promedio del tiempo que toma el recorrido desde un punto hasta otro, como lo muestra la Tabla 1. Esta tabla también permite conocer el estado de cada trayecto en tiempos, y el total de pedidos repartidos al día. De esta forma, para el caso de Y-A, la empresa empleó un total de 9,89 minutos en promedio para llevar la mercancía en los 9 días que se hicieron despachos a este destino (D1, D2, D3, D6, D8, D10, D11, D12, D15). En el caso del último registro, recorrido distancia D-E, la empresa empleó en promedio $14^{\prime}$ minutos y $75^{\prime \prime}$ segundos en esta parte de la ruta. De manera vertical aparece el total de recorrido por días, donde el día 1, la empresa realizó 5 entregas; el día 2, realizó 4; el día 3, 5; (..) el día 13, 5; el día 14,4 y el día 15 se realizaron 5 entregas. En el análisis crítico, se identificaron y clasificaron las rutas actuales, a partir de la Tabla 2; mostrándose ordenados los recorridos de cada ruta diariamente. La tabla 2, muestra el orden de cada ruta por entrega, los destinos en los cuales se debe hacer la entrega y el total de entregas realizadas en el periodo en cada ruta. En las rutas se plantea cada entrega en función del origen y el destino. Así por ejemplo, el día 1 (D1) la ruta 1, comienza la primera entrega en Y (CEDI) y termina en A [Y-A]; el segundo recorrido empieza por $A$ (destino del primer recorrido) y su destino es $D$ [A-D]; la tercera entrega de la ruta 1 comienza en $D$ y su destino es $B$ [D-B]; la cuarta entrega de la ruta 1 en el día 1, (D1), comienza en $B$ y su destino es $E[B-E]$. En total se cumplen 4 entregas en esa ruta el día 1 (D1).

Tabla 2: Ordenamiento de las Entregas por Rutas.

\begin{tabular}{|c|c|c|c|c|}
\hline Días & Ruta 1 & Entregas & Ruta 2 & Entregas \\
\hline $\mathrm{D}-1$ & {$[\mathrm{Y}-\mathrm{A}][\mathrm{A}-\mathrm{D}][\mathrm{D}-\mathrm{B}][\mathrm{B}-\mathrm{E}]$} & 4 & {$[\mathrm{Y}-\mathrm{C}]$} & 1 \\
\hline D-2 & {$[\mathrm{Y}-\mathrm{C}][\mathrm{C}-\mathrm{D}]$} & 2 & {$[Y-A][A-E]$} & 2 \\
\hline D-3 & {$[Y-C][C-B][B-A]$} & 3 & {$[Y-A][A-D]$} & 2 \\
\hline D-4 & [Y-B][B-D] & 2 & & 0 \\
\hline D-5 & {$[Y-D][D-E][E-A][A-B]$} & 4 & & 0 \\
\hline D-6 & {$[Y-A][A-C][C-D]$} & 3 & {$[\mathrm{Y}-\mathrm{E}]$} & 1 \\
\hline D-7 & {$[Y-B][B-E][E-C]$} & 3 & [Y-E] & 1 \\
\hline D-8 & {$[Y-A][A-C][C-E]$} & 3 & [Y-D] & 1 \\
\hline D-9 & {$[Y-C][C-E][E-D]$} & 3 & {$[\mathrm{Y}-\mathrm{D}][\mathrm{D}-\mathrm{A}]$} & 2 \\
\hline D-10 & {$[Y-E][E-D][D-A]$} & 3 & {$[\mathrm{Y}-\mathrm{A}][\mathrm{A}-\mathrm{C}]$} & 2 \\
\hline$D-11$ & {$[Y-A][A-E]$} & 2 & [Y-D] & 1 \\
\hline $\mathrm{D}-12$ & {$[Y-A][A-D][D-C]$} & 3 & {$[\mathrm{Y}-\mathrm{C}]$} & 1 \\
\hline $\mathrm{D}-13$ & {$[\mathrm{Y}-\mathrm{E}][\mathrm{E}-\mathrm{D}]$} & 2 & {$[\mathrm{Y}-\mathrm{B}][\mathrm{B}-\mathrm{C}][\mathrm{C}-\mathrm{A}]$} & 3 \\
\hline$D-14$ & {$[Y-C][C-B][B-A]$} & 3 & {$[Y-D]$} & 1 \\
\hline D-15 & {$[\mathrm{Y}-\mathrm{E}][\mathrm{E}-\mathrm{C}][\mathrm{C}-\mathrm{B}]$} & 3 & {$[Y-A][A-D]$} & 2 \\
\hline & Total Entregas R1 & 43 & Total Entregas R2 & 20 \\
\hline
\end{tabular}


Se registraron 15 y 13 recorridos en las rutas 1 y 2 respectivamente; los días 4 y 5 se registra una ruta. Los días de mayor número de entregas son el D1, D3, D9, D10, D13, D15 con 5 repartos. El D4 se registró 2 entregas. La mayor cantidad de entregas en la ruta 1 se observa en D1 y D5, con 4 repartos. La ruta 2 registró mayor cantidad de entregas en el D13 (3 entregas). Los demás días esta ruta cubre 102 entregas diarias. En el día 12, se hacen dos repartos a un mismo cliente (destino C). En el rutero 1, se observa que el recorrido del día 12 lo termina en el cliente C [D-C], mientras la Ruta 2 realizó ese día una entrega única a C [Y-C]. Se observó, además, que la ruta 2 los días D1, D6, D7, D8, D11, D12, D14, realizó el recorrido hacia un solo destino de distribución. Actualmente la empresa presenta indicadores negativos de cumplimiento en algunas entregas realizadas. En parte se debe a que el criterio escogido al momento de diseñar y empezar la ruta es la distancia geográfica de cada cliente con respecto al CEDI o empresa. Algunos pedidos que se debieron entregar en la mañana, en ocasiones son entregados por la tarde. El análisis de la situación actual del sistema de distribución permitió registrar los tiempos entre los puntos de origen y destino de las rutas, en una gráfica propuesta para este fin, denominada Modelo para estimar tiempos de entrega (en adelante METDE), presentado en la figura 2.

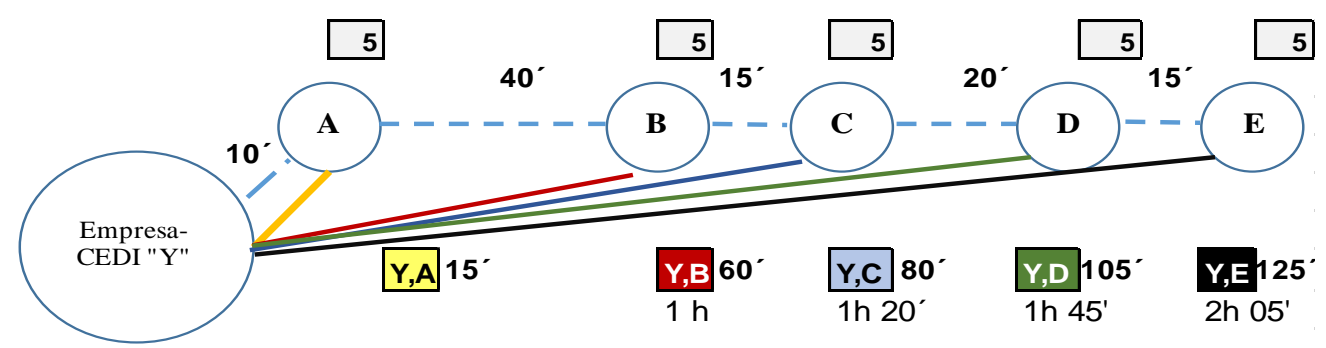

Fig. 2: Modelo para Estimar Tiempos de Entrega (METDE).

El modelo METDE, es la representación gráfica de los tiempos empleados en recorrer las distancias entre la empresa o CEDI y cada uno de los destinos, adicionando el promedio que toma el recibo y verificación de la mercancía (en adelante PRVM). Puede utilizarse para estimar los tiempos entre la empresa y cada punto de entrega, en un rutero con clientes que se encuentran en un mismo sector de la ciudad. Una vez graficados los tiempos de los recorridos entre empresa y los destinos, se procedió con la formulación matemática mostrada en la ecuación 1, para comprobar la exactitud de los cálculos del modelo METDE. La formulación muestra el promedio de tiempos cronometrados desde el origen ( $Y$ ) hasta cada destino, teniendo en cuenta, el tiempo promedio que toma el recibo y verificación de la mercancía (en adelante PRVM) por parte del cliente, para el caso calculado en 5 minutos. Para calcular el PRVM se tiene en cuenta la verificación de la caja (no se apertura) y la firma del acuse de recibo o documento de recibo a satisfacción. EI PRVM puede calcularse registrando el tiempo que toma la verificación de la entrega, teniendo en cuenta las políticas de recibo del cliente, la naturaleza de la mercancía, el proceso al descargar y la importancia del cliente.

Recorrido $Y$ hasta $A,[Y-A]=[(Y-A)+(1 \times P R V M)]=\left[\left(10^{\prime}\right)+(1 \times 5)\right]=15^{\prime}$ minutos.

Recorrido $Y$ hasta $B,[Y-B]=[((Y-A)+(A-B))+(2 \times P R V M)]=\left[\left(\left(10^{\prime}\right)+\left(40^{\prime}\right)\right)+(2 \times 5)\right]=60^{\prime}$ minutos.

Recorrido $Y$ hasta $C,[Y-C]=[((Y-A]+(A-B)+(B-C))+(3 \times P R V M)]=\left[\left(\left(10^{\prime}\right)+\left(40^{\prime}\right)+\left(15^{\prime}\right)\right)+(3 \times 5)\right]=$

80'minutos.

Recorrido Y hasta D, [Y-D] $=[((Y-A)+(A-B)+(B-C)+(C-D))+(4 x P R V M)]=\left[\left(\left(10^{\prime}\right)+\left(40^{\prime}\right)+\left(15^{\prime}\right)+\left(20^{\prime}\right)\right.\right.$

$+(4 \times 5)]=105^{\prime}$ minutos.

Recorrido Y hasta $E,[Y-E]=\left[((Y-A)+(A-B)+(B-C)+(C-D)+(D-E)+(5 x P R V M)]=\left[\left(\left(10^{\prime}\right)+\left(40^{\prime}\right)+\left(15^{\prime}\right)\right.\right.\right.$

$\left.+\left(20^{\prime}\right)+(15)+(5 \times 5)\right]=125^{\prime}$ minutos.

Se interpreta que desde $Y$ hasta $A(Y-A)$, en promedio el recorrido toma 10' minutos; de $Y$ hasta $B(Y-B)$ : 50' minutos; de $Y$ hasta $C(Y-C): 65^{\prime}$ minutos; de $Y$ hasta $D(Y-D): 85^{\prime}$ minutos; de $Y$ hasta $E(Y-E): 100^{\prime}$ minutos. Cálculos aproximados realizados a partir de la Tabla 1, los cuales son trasladados al modelo para estimar tiempos de entrega (METDE).

En la tabla se observa que se agrega un promedio de $5^{\prime}$ minutos (PRVM), lo cual significa que desde $Y$ hasta A la empresa necesitó en promedio, 15 minutos para hacer el recorrido y la entrega del producto a ese destino: $[\mathrm{Y}-\mathrm{A}]: 10^{\prime}+5^{\prime}=15^{\prime}$ minutos; desde $[\mathrm{Y}-\mathrm{B}]: 50^{\prime}+2[5]=60^{\prime}$ minutos. Para este recorrido $[\mathrm{Y}-\mathrm{B}]$, se multiplicó el PRVM ( 5 'minutos) por 2, ya que se realizaron dos paradas para entregar mercancía en cada destino (A y B). De esa forma se procedió con los demás destinos, estimando un total de $125^{\prime}$ minutos para el trayecto entre (Y-E), dónde se realizaron 5 paradas para entregar los productos (A-B-C-D-E). La fórmula de este trayecto queda 
expresada: $[\mathrm{Y}-\mathrm{E}]=\left[10^{\prime}+40^{\prime}+15^{\prime}+20^{\prime}+15^{\prime}+\left(5 \times 5^{\prime}\right)\right]$. Estos tiempos se muestran en el modelo METDE (figura 2 ), ordenados por recorrido. En los cuadros en la parte superior del modelo METDE, se consignan los 5 'minutos estimados de tiempo promedio de recibo y verificación de la mercancía (PRVM). En la Tabla 3 se muestra el análisis crítico cualitativo. Se identificaron variables críticas en la(s) ruta(s) de distribución que dificultan el normal desarrollo de la distribución. También se encontraron quejas de los clientes por entregas tardías, toda vez que se promete la entrega durante el "trascurso de la mañana" o "de la tarde" incumpliendo en ocasiones, lo cual disminuye el índice de satisfacción de los clientes.

Tabla 3: Variables Críticas en la Distribución en el Entorno de Barranquilla.

\begin{tabular}{|r|l|}
\hline 1 & Tráfico vehicular \\
\hline 2 & Inseguridad del acceso \\
\hline 3 & Mantenimiento y reparación de vías públicas \\
\hline 4 & Mantenimiento y reparación de acometidas de servicios públicos \\
\hline 5 & accidentes en la ruta de reparto \\
\hline 6 & accidente del vehículo de reparto \\
\hline 7 & Disponibilidad y Disposición del cliente para recibir mercancía \\
\hline 8 & Disponibilidad de vehículos públicos para reparto \\
\hline 9 & Variación de tarifas en fechas especiales (24 de diciembre) \\
\hline 10 & Estado de las vías Públicas \\
\hline 11 & Daños \\
\hline 12 & Construcciones a los lados de las Vías \\
\hline 13 & Altura de cables eléctricos \\
\hline 14 & Cercanía en la ruta de distribución de cementerios o funerarias (cortejos fúnebres). \\
\hline
\end{tabular}

Algunas de estas variables críticas no son frecuentes, otras se vuelven frecuentes durante el recorrido, dependiendo de la duración de una obra de mantenimiento, del acceso a puntos clave de la ciudad, entre otras. La propuesta de mejoramiento, (paso 3) consistió en plantear soluciones para cumplir con los tiempos de pactados de las entregas, aumentando la satisfacción de los clientes a través de ruteros programados por horarios con el fin de llegar a los clientes en la hora acordada. La propuesta se implementa a partir del día 16, programando 4 pedidos a los clientes A, B, C y E en los horarios acordados en la Tabla 4.

Tabla 4: Horarios de Entrega Día 16 (D16).

\begin{tabular}{|c|c|}
\hline Destino & Horario de Entrega \\
\hline B & $8: 15$ \\
\hline C & $8: 20$ \\
\hline A & $9: 00$ \\
\hline E & $9: 40$ \\
\hline
\end{tabular}

En la Tabla 5, se plantearon las rutas especificando el inicio, el horario y el tiempo en el cual debe iniciar cada ruta, también se distingue el orden de la entrega. Posteriormente se hace una estimación para el cumplimiento de la ruta, dónde se decide si se programa o no el recorrido de acuerdo con el cumplimiento de los horarios pactados con el cliente. La ruta [Y-B] debe empezar a las 7:25, ya que el tiempo promedio del recorrido entre $Y$ y el destino $B$ es de 50'minutos (señalados en el modelo METDE). El resultado fue obtenido al restar del horario de la entrega, el tiempo en promedio del recorrido. El recorrido entre los destinos [B-C] empezaría a las 8:20 para la ruta 1 , resultado obtenido al sumar al horario de entrega en cliente $B(8: 15)$, los 5 minutos del recibo y verificación de mercancías (PRVM). Si a este resultado se le suman 15 minutos que toma en promedio el recorrido entre los destinos B-C (indicados en el modelo METDE), el resultado es 8:35, horario mayor al horario programado para la entrega del cliente $C$, por lo que no se programó el recorrido de la ruta 1 hasta el destino $\mathrm{C}$, ya que resultaría en una demora de 15 minutos en el tiempo pactado.

En el caso del recorrido entre los destinos [B-A] se logró programar su entrega, ya que la estimación arrojó un tiempo igual al horario de entrega, lo cual resultaría en una entrega a tiempo, tal como se indica en la tabla 5 (recorrido B-A). Para el diseño de la ruta 2, se procedió bajo el mismo razonamiento, quedando diseñadas las rutas de acuerdo con la tabla 5 de la siguiente forma: Ruta 1: [Y-B] [B-A]; Ruta 2: [Y-C] [C-E]. Para ambas rutas se cumplen las restricciones de tiempos y horarios, por lo cual se afirma que se logran las entregas según los horarios establecidos. 
Tabla 5: Diseño de las Rutas.

\begin{tabular}{|c|c|c|c|c|c|}
\hline \multirow{2}{*}{$\begin{array}{l}\text { D 16 } \\
\text { Planteamiento }\end{array}$} & \multicolumn{2}{|l|}{ Ruta 1} & \multicolumn{3}{|c|}{ Ruta 2} \\
\hline & Ruta: $[\mathrm{Y}-\mathrm{B}][\mathrm{B}-\mathrm{A}]$ & & Planteamiento & Ruta: [Y-C][C-E] & \\
\hline Inicio de Ruta. & $\begin{array}{l}{[Y-B] 8: 15-50=7: 25} \\
\text { Se programó, el recorrido inicia a } \\
\text { las 7:25 }\end{array}$ & Entrega 1 & Inicio de Ruta. & $\begin{array}{l}{[Y-C] 8: 20-65=7: 15} \\
\text { Se programó, el recorrido inicia a } \\
\text { las } 7: 15\end{array}$ & Entrega 1 \\
\hline $\begin{array}{l}\text { Estimación } \\
\text { Recorrido [B- } \\
\text { C] }\end{array}$ & $\begin{array}{l}8: 15+5=8: 20 \\
\text { [B-C] 8:20+15 = 8:35 > 8:20. } \\
\text { No se Programa por exceder en } \\
15 \text { minutos el horario de entrega. }\end{array}$ & $\begin{array}{l}\text { No se } \\
\text { Programa }\end{array}$ & $\begin{array}{l}\text { Estimación } \\
\text { Recorrido [C- } \\
\text { E] }\end{array}$ & $\begin{array}{l}\text { 8:20+5=8:25; [C-E] } 8: 25+35= \\
9: 00<9: 40 \text { Se Programó. }\end{array}$ & Entrega 2 \\
\hline $\begin{array}{l}\text { Estimación } \\
\text { Recorrido [B- } \\
\text { A] }\end{array}$ & $\begin{array}{l}\text { 8:15+5=8:20; [B-A] 8:20+40 = } \\
\text { 9:00 =9:00. Se procedió a } \\
\text { programar ya que el tiempo del } \\
\text { recorrido es igual al del horario de } \\
\text { entrega. }\end{array}$ & Entrega 2 & & & \\
\hline $\begin{array}{l}\text { Tiempo } \\
\text { Estimado }\end{array}$ & $.=[50]+[40]+[2(5)]=100^{\prime}$ & & \begin{tabular}{|l|} 
Tiempo \\
Estimado \\
\end{tabular} & $.=[65]+[35]+[2(5)]=110^{\prime}$ & \\
\hline & Total Entregas & 2 & & Total Entregas & 2 \\
\hline
\end{tabular}

\section{DISCUSION FINAL}

En el departamento del Atlántico, existen clústeres logísticos dónde la cadena de valor concentra sus esfuerzos en mejorar la calidad del servicio a través de la reducción y eficiencia de los costos globales, con un especial interés en optimizar la logística de transporte (Salas et al., 2016). El método METDE parte de la base de mejorar la distribución en cuanto a condiciones de tiempo pactadas con el cliente, con el cumplimiento como propósito principal. Entre las principales limitaciones de la metodología METDE, a diferencia de otros modelos propuestos, es que el diseño de rutas es propuesto en función del criterio cumplimiento de horario, aún por encima de la importancia de la distancia geográfica entre los puntos. Metodologías como la del "procedimiento para el diseño de redes de distribución logística", proponen la optimización del sistema de transporte a partir de 5 fases para hallar una solución óptima de transporte, tiene como principal criterio de desempeño los costos del sistema de distribución (Ortiz et al., 2014).

Un propósito común con el modelo para el enrutamiento de vehículos basado en la metaheurística bacteriana (Amador et al., 2014) consiste en que el método METDE propone resolver el enrutamiento de vehículos asignados a rutas, para el caso del METDE, a través de la minimización de estas y su distancia total de recorrido; integrando las operaciones a partir de tecnologías de información y comunicación, diseñadas en este caso, a través del aplicativo de Excel, teniendo en cuenta que las capacidades de las tecnologías de información y comunicaciones tienen efectos significativos sobre la integración logística (Prajogo y Olhager, 2012). La comunicación es una de las condiciones básicas de la gestión que potencia el intercambio de información (Henríquez et al., 2018). La información es pieza clave en la logística de salida y la comunicación permite coordinar e integrar a los actores y recursos según lo planeado, por ello, la implementación del método METDE resultará más eficiente al ser acompañada de información inmediata, diaria y precisa sobre los pedidos, destinos y tiempos pactados con los clientes. La metodología METDE, brindará la posibilidad de mejorar la eficiencia y calidad en el servicio al momento de entregar las mercancías en los tiempos prometidos a los clientes, permitiendo que la empresa innove en la prestación de sus servicios (Cardona et al., 2017).

A diferencia del modelo CVRPfC (Problema de enrutamiento de vehículos con capacidad para transportistas), el METDE no propone la competitividad de la empresa por la vía de los costos. El modelo CVRPfC proponer mejorar la competitividad de operadores proporcionando mejores tarifas a sus clientes, reduciendo los costos de distribución (Rojas et al., 2018). Considerando factores del contexto logístico, el modelo para redes colaborativas Non-for-profit (Balza-Franco et al., 2017) para cadenas de suministro, no considera las eventualidades que pueden ocurrir en el transporte, tales como fallas de infraestructura. La metodología METDE, sí es incluyente de variables críticas muy propias de la distribución en Barranquilla, Colombia. El tráfico vehicular, la inseguridad del acceso, mantenimiento y reparación de vías públicas y de acometidas de servicios públicas, así como el estado de las vías públicas, son algunas de las variables consideradas al momento del diseño de las rutas. La metodología METDE al igual que las nuevas tendencias para el monitoreo de la Cadena de suministro, es un sistema o aplicativo que soporta sus bases en un enfoque tecnológico de sistemas de información a partir de la interconexión de información con una base de datos (histórico de las rutas) a partir de software y hardware (Badia-Melis et al., 2018).

Para el METDE la eficiencia de la logística de transporte se relaciona estrechamente con la variable tiempo, por lo que se considera importante realizar un estudio más a fondo sobre los factores que componen el tiempo promedio que toma el recibo y verificación de la mercancía (PRVM). 


\section{CONCLUSIONES}

De acuerdo con el trabajo presentado y a los resultados obtenidos, se pueden plantear las siguientes conclusiones principales:

1.- La metodología METDE ha probado ser una herramienta que apoya la planificación de rutas de distribución diseñadas para cumplir con los tiempos acordados con los clientes, cuando el criterio de ordenamiento obedece a la distancia del destino con respecto a la empresa o centro de distribución y el respeto de los horarios. En la tabla 5, se observa la planificación de las rutas para el día 16, bajo el criterio del cumplimiento de horario.

2.- La Tabla 5 puede ser empleada como una herramienta para organizar las rutas y evitar sobrecostos o retrasos en las entregas, apoyándose en el modelo METDE para estimar los cálculos de los tiempos al trazar las rutas.

3.- Las rutas deben programarse evitando situaciones como el día 12, dónde se visita dos veces al mismo cliente $(C)$ en el día, lo cual representa sobrecostos para la empresa al no optimizar la capacidad del vehículo en ruta. A no ser que se trate de un pedido extra para el cual se tenga disponibilidad de inventario, o del tratamiento de un cliente especial de la empresa, el pedido debe aplazarse para el día siguiente a su recepción.

4.- El cálculo del PRVM reviste relevancia para el modelo propuesto en la medida en que afecta los tiempos de las rutas. Las directivas deben estimar el tiempo teniendo en cuenta las políticas de recibo del cliente, la naturaleza de la mercancía (peso, tamaño, necesidad de chequeo físico); el proceso al momento de descargar (mecanización del procedimiento por herramientas, equipos o vehículos para carga pesada), así como la importancia de cada cliente a nivel financiero (clientes paretto). Se deben identificar las variables críticas que afectan la distribución de manera contextual, ya que cada entorno es diferente en infraestructura, cultura de manejo, acceso a vías, así como el uso del suelo alrededor de los clientes o la empresa.

\section{REFERENCIAS}

Amador, J., C. Paternina y J. Montoya, Solving the heterogeneuos vehicle routing problema with the time Windows and multiple products via a bacterial meta-heuristic, doi: https://doi.org/10.1504/IJAOM.2014.059622, Int. J. Advanced Operations Management, 6(1), 81-100 (2014)

Amaya, J., C. Viloria y A. Santander, Diseño de cadena de suministros resilientes, Editorial U. del Norte, Colombia (2014) Arrubla, J., Notas de Fundamento de Mercadeo, Publicaciones Congregación Mariana, Colombia (2011)

Badia-Melis, R., U. Mc Carthy y otros tres autores, New trends in cold chain monitoring applications - A review, doi: 10.1016/j.foodcont.2017.11.022, Food Science \& Technology, 86, 170-182 (2018)

Balza, V., C. Paternina y otros tres autores, A Collaborative Supply Chain Model for Non-for-Profit Networks Based on Cooperative Game Theory, doi: https://doi.org/10.1504/IJLSM.2017.082614, International Journal of Logistics Systems and Management, 26(4), 475-496 (2017)

Cardona, D., V. Balza-Franco y G. Henríquez, Innovación en el sector de los servicios: aproximación conceptual y revisión de su aporte a la economía, Revista Espacios, ISSN: 07981015, 38 (21), 36 (2017)

Cardona, D., V. Balza y G. Henríquez, Innovación en los procesos logísticos: retos locales frente al desarrollo Global, Editorial Universidad Libre, Cartagena, Colombia (2017)

Cardona, D., G. Henríquez y otros tres autores, Endomárketing: Estrategia de innovación en las organizaciones en el siglo XXI, Revista Espacios, ISSN: 07981015, 38(57), 26 (2017)

Casanava, P., SIG per gestionar rutes en camins no cartografiats, Tesis de Pregrado, Universitat Oberta de Catalunya, España (2010)

Chen, W., H. Cgai, Q. Wang y H. Hong, Landslide susceptibility mapping based on GIS and support vector machine models for the Qianyang County, China, doi: https://doi.org/10.1007/s12665-015-5093-0, Environ Earth Sci, 75(6), 1-13 (2016)

Cho, D., Y. Lee, S. Ahn y M. Hwang, A framework for measuring the performance of service supply chain management, doi: https://doi.org/10.1016/j.cie.2011.11.014, Computers \& Industrial Engineering, 62(3), $801-818$ (2012)

Falcón, O., M. Petterson, S. Benavides e I. Sarmenteros, Los métodos cuantitativos en la mejora de los procesos del catering, Ingeniería Industrial, ISSN: 1815-5936, 37(1), 70-77 (2016)

Frohlich, M. y R. Wetbrook, Arcs of Integration: an International Study of Supply Chain Strategies, doi: https://doi.org/10.1016/S0272-6963(00)00055-3, Journal of Operations Management, 19(2), 185-200 (2011)

Gómez, R. y A. Correa, Análisis del Transporte y Distribución de Materiales de Construcción Utilizando Simulación Discreta en 3D, Boletín de Ciencias de la Tierra, ISSN: 0120-3630, (30), 39-52 (2011)

Gutierrez, V., J. Palacio y J. Villegas, Reseña del Software disponible en Colombia para el diseño de rutas de distribución y servicios, Revista Universidad Eafit, ISSN: 0120-341X, 43(145), 60-80 (2007) 
Hasan, B., N. Zulkifli, S. Nizaroyani y A. Malak, Review of supply chain performance measurement systems: 1998-2015, doi: https://doi.org/10.1016/j.compind.2016.07.002, Computers in Industry, 82, 135-150 (2016)

Henríquez-Fuentes, G., J. Lombana-Coy y otros seis autores, La gobernanza y su relación con la competitividad en una firma integrada a una cadena de suministro, Revista Espacios, ISSN: 0798-1015, 39(18), 27 (2018)

Henríquez, G., F. Vallaeys y M. Garzón, El aprendizaje organizacional como herramienta para la universidad que aprende a ser responsable socialmente, doi: http://dx.doi.org/10.21803\%-2Fpenamer.11.20.499, Revista Pensamiento Americano, 11(20), 116-140 (2018)

Henríquez, G., Investigación cualitativa en modelos de gestión logística y sus estrategias de desarrollo en la costa Caribe colombiana, Ad-gnosis, ISSN: 2344-7516, 2(2), 65-85 (2013)

Henríquez, G., J. Rada y A. Torrenegra, Medición de variables psicológicas, económicas y sociales para identificar factores ocultos, doi: http://dx.doi.org/10.17981/econcuc.37.1.2016.08, Revista Económicas CUC, 37(1), 177-202 (2016)

Jhawar, A., S. Garg y S. Khera, Analysis of the Skilled Work Force Effect on the Logistics Performance Index- Case Study from India, doi: DOI 10.1007/s12159-014-0117-9, Logistics Research, 7(1), 1-10 (2014)

Kaplan, R. y D. Norton, Using the balanced scorecard as a strategic management system; Harvard business review, ISSN: 0017-8012, 74(1), 75-85 (1996)

Kolli, S., P. Damodaran y G. Evans, Geographic information system based decision support systems for facility location, routing, and scheduling, doi: https://doi.org/10.1016/0360-8352(93)90298-C, Computers and Industrial Engineering, 25(14) 369-372 (1993)

Kovacs, A., B. Golden, R. Hartl y S. Parragh, The Generalized Consistent Vehicle Routing Problem, doi: https://doi.org/10.1287/trsc.2014.0529, Transportation Science, 49(4), 796-816 (2015)

Kozlenkova, I., G. Hult y otros tres autores, The Role of Marketing Channels in Supply Chain Management, doi: https://doi.org/10.1016/j.jretai.2015.03.003, Journal of Retailing, 91(4), 586-609 (2015)

León, J., E. González, J. Díaz y J. Sorlí, Desarrollo de una herramienta de gestión de flotas de vehículos eléctricos, Economía Industrial, ISSN: 0422-2784, 400, 59-68, (2016)

Martínez, J., J. De la Hoz, G. García e I. Molina, Gestión logística en Pymes del sector de operadores de carga del departamento del Atlántico, Revista espacios, ISSN: 0798-1015, 38(58), 13 (2017)

Mydin, M., A. Othuman y K. Jie, Construction waste material management and logistic system, Analele Universitatii' Murgu, ISSN: 1453-7397, 21(1), 220-230 (2014)

Ortiz, M., R. Rodríguez y O. Coronado, Propuesta de un Sistema de distribución para la cadena de suministro de la empresa Pastor, C.A., Revista Espacios, ISSN: 0798-1015, 35(6), 20 (2014)

Prajogo, D. y J. Olhager, Supply chain integration and performance: The effects of long-term relationships, information technology and sharing, and logistics integration, doi: https://doi.org/10.1016/j.ijpe.2011.09.001, International Journal of Production Economics, 135(1), 514-522 (2012)

Rajeev, A., R. Pati, S. Pahdi y K. Govindan, Evolution of sustainability in supply chain management: A literature review, doi: https://doi.org/10.1016/j.jclepro.2017.05.026, Journal of Cleaner Production, 162, 299-314 (2017)

Rojas-Cuevas, I., S. Caballero-Morales, J. Martínez-Flores y J. Mendoza-Vazquez, Capacitated vehicle routing problem model for carriers, doi: https://doi.org/10.4102/jtscm.v12i0.345, J. of Transport and Supply Chain Management, 12(0), 1-9 (2018)

Salas, K., C. Acosta y otros tres autores, Análisis estratégico de cluster de servicios logísticos, Revista Espacios, ISSN: 0798-1015, 37(28), 5, (2016)

Suarez-Chilma, V., W. Sarache e Y. Costa, Una Solución al Enrutamiento de Vehículos en Ciudades Montañosas Considerando Aspectos Ambientales y Económicos, doi: http://dx.doi.org/10.4067/S0718-07642018000300003, Información Tecnológica, 29(3), 3-14 (2018)

Vélez, T., Logística empresarial, Gestión eficiente del flujo de suministros, Ediciones de la U., Colombia (2014)

Vidal, C., J. Londoño y F. Contreras, Aplicación de Modelos de Inventarios en una Cadena de Abastecimiento de Productos de Consumo Masivo con una Bodega y N Puntos de Venta, Ingeniería y competitividad, ISSN: 0123-3033, 6(1), 35-52 (2004)

Voznenko, N. y T. Roma, Features of logistic system adaptive management, CES Working Papers, ISSN: 2067-7693, VII (2), 357-364 (2015)

Wang, Y., S. Wallace, B. Shen y Choi, T., Service supply chain management: A review of operational models, doi: https://doi.org/10.1016/j.ejor.2015.05.053, European Journal of Operational Research, 247(3), 685-698 (2015)

Ying, K. y S. Lin, Minimizing makespan for the distributed hybrid flowshop scheduling problem with multiprocessor tasks, doi.org/10.1016/j.eswa.2017.09.032, Expert systems with applications, 92, 132-141 (2018)

Yu, F., T. Kaihara y otros tres autores, A multi-attribute multi-item negotiation mechanism of supply chain networks between buyers and Sellers, doi: https://doi.org/10.1080/00207543.2015.1034328, International Journal of Production Research, 53(20), 6143-6160 (2015) 